\title{
Assessment of the Depth of Anesthesia based on Symbolic Dynamics of the EEG
}

\author{
Nadine Tupaika, Montserrat Vallverdu, Mathieu Jospin, Erik W. Jensen, Member, IEEE, \\ Michel M. R. F. Struys, Hugo E. M. Vereecke, Andreas Voss, Pere Caminal
}

\begin{abstract}
Methodologies based on symbolic dynamics have successfully demonstrated to reflect the nonlinear behavior of biological signals. In the present study, symbolic dynamics was applied to the electroencephalogram (EEG) in order to describe the level of depth of anesthesia. The EEG was transformed to symbol sequences. Words of three symbols were built from this symbolic series. The results obtained from the EEGs of 36 patients undergoing anesthesia showed that the probabilities of the word types were able to reflect the depth of anesthesia in a similar way to the auditory evoked potential index AAI, a commercial index.
\end{abstract}

\section{INTRODUCTION}

$I^{\prime}$ $\mathrm{t}$ has been reported that a too deep anesthesia, apart from increasing drug consumption, can cause longer recovery time $[1,2,3]$. On the other hand, too low drug concentration may lead to perioperative awareness [4]. Therefore, it is a relevant challenge to monitor the depth of anesthesia (DOA) reliably. Current research in DOA assessment is focused on noninvasive monitoring of the Electroencephalogram (EEG) as the electrical activity of the brain is affected by the administration of anesthetic agents [5].

Two main techniques have been introduced in clinical practice: the study of auditory evoked potentials (AEP) [6,7] which evaluates the response to an auditory stimulation, and the analysis of the spontaneous EEG to derive composite indexes such as bispectral index (BIS) $[8,9]$ for example, which studies the coupling between different frequencies of the Fourier spectrum.

Observations have suggested that biological signals like the EEG present nonlinear behavior [10]. Therefore, it is necessary to apply methods that study this nonlinear information. Symbolic dynamics analysis, one of these nonlinear methods, has been introduced successfully to characterize the electrocardiogram in heart rate variability studies [11]. As the complexity of the EEG decreases with

Manuscript received April 22, 2010. This work was supported within the framework of the CICYT grant TEC2004-02274 from the Spanish Government. N. Tupaika, M. Vallverdú, M. Jospin, E. W. Jensen and P. Caminal are with Dept. ESAII, Centre for Biomedical Engineering Research, Universitat Politècnica de Catalunya, CIBER of Bioengineering, Biomaterials and Nanomedicine (CIBER-BBN), Barcelona, Spain; email: montserrat.vallverdu@upc.edu. M.M.R.F. Struys is with the Department of Anesthesiology, University Medical Center and University of Groningen, Groningen, The Netherlands and with the Department of Anesthesia, Ghent University Hospital, Gent, Belgium. H.E.M. Vereecke is with the Department of Anesthesia, Ghent University Hospital, Gent, Belgium. A. Voss is with the University of Applied Sciences Jena, Department of Medical Engineering, Jena, Germany. anesthesia [12], we theorized that this approach is also able to reflect the DOA changes. The aim of this study is thus to study the application of Symbolic Dynamics to the EEG, as a noninvasive DOA measure.

\section{MATERIALS AND METHODS}

\section{A. Data Recording and Pre-processing}

With the approval of institutional ethics committee written informed consent was obtained in a first database from 19 women and in a second database from 17 women, with 18 to 60 year old, scheduled for ambulatory gynecological surgery in the Ghent University Hospital, Belgium. The patients belonged to the ASA I group (ASA, American Society of Anesthesiology), corresponding to the minor risk level of a 1-to-5 scale (increasing risk). Exclusion criteria were a weight less than $70 \%$ or more than $130 \%$ of ideal body weight, neurological disorders, and recent use of psychoactive medication, as well as alcohol.

All patients received continuous infusion of propofol fixed at $300 \mathrm{ml} / \mathrm{h}$ by a computer-assisted infusion device (RUGLOOP $[13,14]$ ). The drug concentration in the central nervous system was reflected by the effect-site concentration which was obtained by using a three-compartment pharmacokinetic-dynamic model enlarged with an effect-site compartment $[15,16]$. To yield a time to peak effect, the effect-site concentration was computed $1.6 \mathrm{~min}$ after bolus injection $[17,18]$. Propofol was infused trough a large left forearm vein. Before starting the drug administration, all patients were asked to close their eyes and relax for $2 \mathrm{~min}$. The infusion was continued until reaching deep anesthesia, when EEG showed a characteristic behavior called burst suppression, consisting of periods of high amplitude (bursts) followed by periods of very low amplitude (suppression) [8]. Infusion was stopped when the burst suppression ratio of $80 \%$ or higher was achieved, and in case of a mean arterial blood pressure lower than $50 \mathrm{mmHg}$.

The AEP index AAI [6] was calculated using the A-Line monitor (Danmeter A/S, Odense Denmark). Three electrodes were positioned: one at the mid forehead $(+)$, one at the left forehead (reference) and one at the left mastoid (-). All hemodynamic data together with the AAI index were logged automatically every $10 \mathrm{~s}$. The A-Line monitor provided a measure of the burst suppression ratio (BS), which represents the fraction of time where the EEG has small amplitudes. As well, the raw EEG was recorded by the monitor. The sampling frequency was $900 \mathrm{~Hz}$, with a resolution of 16 bits and a recording time between 10 and 15 
min. A traditional way of distinguishing between the different sedation levels is using the MOAA/SS (Modified Observer's Assessment of Alertness/Sedation Scale), an objective assessment of the patient state by the anesthesiologist. Such assessment is not available in this study, so it has to be extracted from the data base. In order to maintain coherence, the indexes from one single technique are considered, the ones provided by the AEPs. Previous studies [6] defined the following states: awake with an AAI index higher than 60, anesthetized with an AAI index lower than 30 and sedated in the case of an AAI index between these two values.

Since burst suppression refers to a deep level of anesthesia the raw EEG was cut-off 10s before its onset. Moreover, the EEG was down sampled to $100 \mathrm{~Hz}$ and filtered with a Butterworth filter of 5-th order with a cut-off frequency of $45 \mathrm{~Hz}$, in order to reduce the influences of the electromyogram and external noise.

\section{B. Symbolic Dynamics}

The analysis of symbolic dynamics proved to be adequate for the study of complex systems and to describe dynamic aspects within time series, as it has been demonstrated in the study of electrocardiogram [11]. The analyzed time series is transformed to symbols from a given alphabet. Detailed information is lost in the transformation but the robust information is preserved and the coarse dynamic behavior can be analyzed $[19,20]$.

On the basis of already evaluated methods [11], four symbols were used to describe the distance of each signal sample to the mean value $\mu$ of the EEG.

If $x_{n}$ is the filtered time series of the EEG with $n$ samples

$$
X=\left\{x^{E E G}\right\}_{n=0,1, . .} x \in \mathbb{R}
$$

it is transformed to symbols

$$
S=\left\{S^{E E G}\right\}_{n=0,1, \ldots} S \in \mathbb{N}
$$

with the following definition

$$
s_{n}= \begin{cases}0: & \mu<x_{n} \leqq(1-a) \mu \\ 1: & (1+a) \mu<x_{n}<\infty \\ 2: & (1-a) \mu<x_{n} \leqq \mu \\ 3: & 0<x_{n} \leqq(1-a) \mu\end{cases}
$$

The factor $a$ was set to 0.05 , in order to match the standard deviation of the EEG. Moreover, to consider 10 minute EEG with a sample frequency of $100 \mathrm{~Hz}$ corresponds to 60000 symbols.

After transforming the filtered signal to a symbol sequence, words of length of three symbols were built with an overlapping of two symbols. In this way, 64 different types of words $w_{i j k}$ were obtained. These words were sorted in a distribution matrix. The methodology of EEG transformation is shown in Fig. 1.

The first database was used for training. For each patient, the value of the AAI defined the actual state of anesthesia in which was distinguished between "awake", "sedated" and "anesthetized". The probability of each word type $p_{w i j k}$ for each patient and each of those states was calculated.

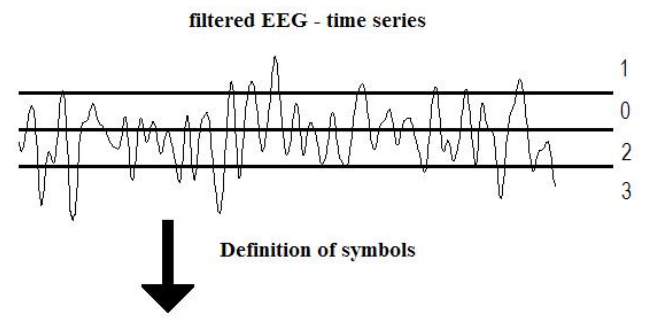

symbol sequence
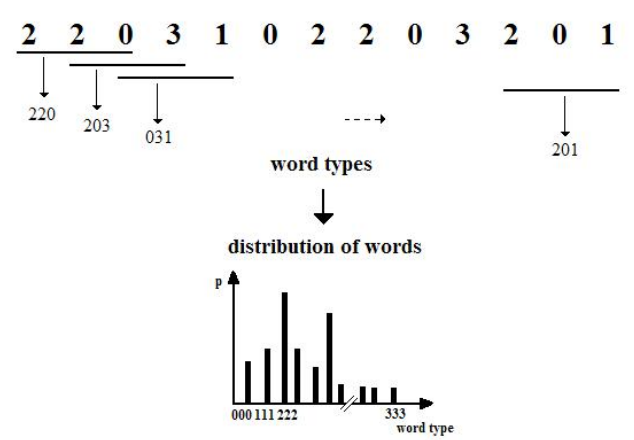

Fig. 1. Methodology of EEG transformation to a symbol series, and how the words are built. The distribution of the 64 different words is shown in a histogram.

Moreover, Shannon entropy $(S H)$ and Rényi entropy $\left(R E_{q}\right)$, as a generalization of the Shannon entropy, were computed in order to investigate the complexity of the EEG in the different states of anesthesia.

$$
\begin{gathered}
S H=-\sum_{z=1}^{N} p_{w_{i j k}}(z) \log _{2}\left(p_{w_{i j k}}(z)\right) \\
R E_{q}=\frac{1}{1-q} \log _{2}\left(\sum_{z=1}^{N} p_{w_{i j k}}^{q}(z)\right)
\end{gathered}
$$

with $q=\{0.1,0.15,0.2,0.25,0.3,0.5,1,2,3,4\}$

$$
i, j, k=\{0,1,2,3\}
$$

In the statistical univariate analysis, the group differences were non-parametrically tested using the Mann-Whitney-UTest and the significance level was provided by the $p$-value. For stratifying and classifying the anesthesia states through the probabilities of the word types, $p_{w i j k}$, a linear discriminant function was applied. By means of these analyses, the words with the best ability to discriminate were selected.

In a second step, those words were used to analyze the first database in a window of 10 s along the EEG to provide the possibility of a real-time application. Finally, the second data base was tested with this 10 s frames as a validation of the method.

To evaluate the correlation between the evolution of the probability and the underlying anesthetic depth (effect-site concentration), the prediction probability $\left(P_{k}\right)$ [21] was applied, as it has been used in recent publications [22-24]. 
Given two randomly selected data points with distinct anesthetic drug concentration, the $P_{k}$ value describes the probability that a parameter correctly predicts which of the data points is the one with higher (or lower) anesthetic drug concentration. A $P_{k}$ value of 1 means that the indicator is perfectly concordant with the control variable, namely this indicator value increases or decreases monotonically with the underlying anesthetic depth; a $P_{k}$ value of 0.5 means that the indicator value is not superior to the value obtained by chance.

In our studies we compared the $P_{k}$ value of the probability of words derived from symbolic dynamics and the AAI in terms of prediction of the Propofol effect-side concentration.
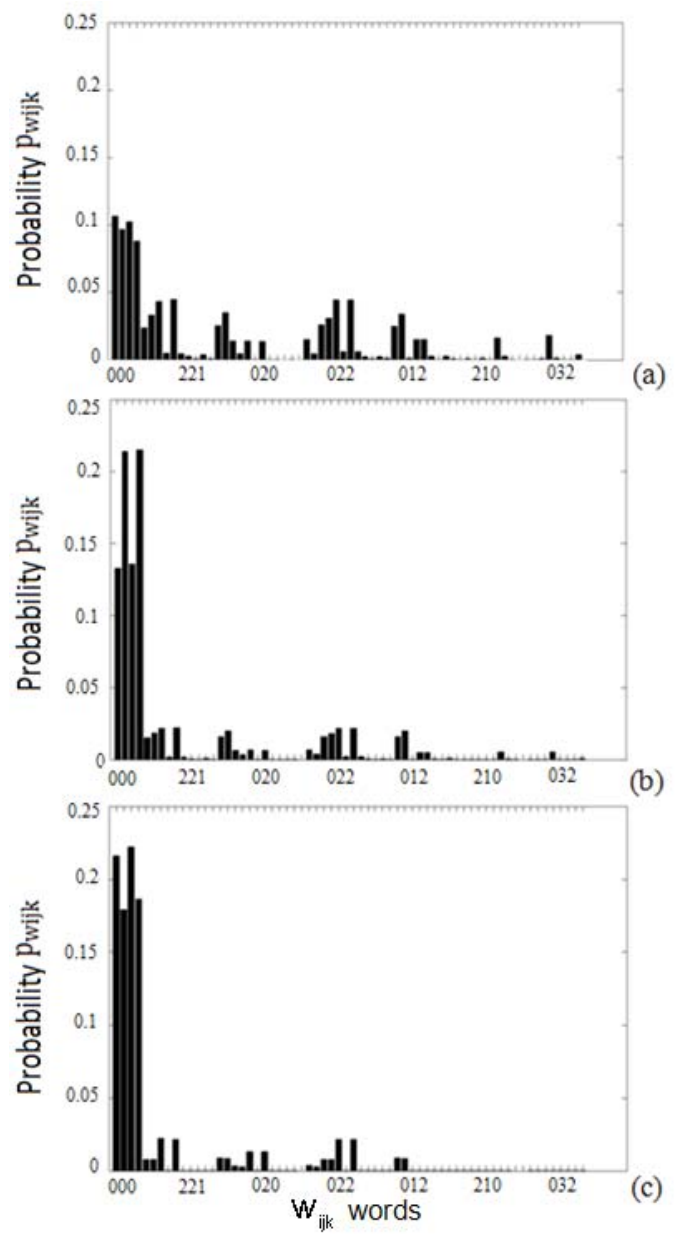

Fig. 2. Histogram of the probabilities of the words is shown for the state: (a) awake; (b) sedated; (c) anesthetized.

\section{RESULTS}

\section{A. Analysis of Complete EEG Recordings}

The accumulated distribution of each state of anesthesia for the whole EEG of the first data base is shown in Fig. 2. It can be observed that the probabilities of the single word types are changing subject to the DOA. This is confirmed by the result of the Mann-Whitney-U-Test that was applied for each word in each state. The mean value and the standard deviation for the best words are reflected in Table I. The $p$ values and the results of the statistical discriminant analysis of selected words are shown in Table II.
The Shannon entropy and Rényi entropy verify the different complexity of the EEG referring to the state of anesthesia (Table III). In this Table, the entropies with the best $p$-values are shown. However, the discriminant analysis demonstrates that the entropies are not able to characterize the states better than the probabilities of the words $p_{w i j k}$. In general, the differentiation between the states "sedated" and "anesthetized" is the most difficult to discriminate.

TABLE I

Mean and Standard Deviation of the Selected Words

\begin{tabular}{llll}
\hline \multicolumn{3}{l}{ mean \pm standard deviation } & \\
\cline { 1 - 3 } words & awake & sedated & anesthetized \\
\hline \hline 000 & $0.111 \pm 0.018$ & $0.063 \pm 0.013$ & \\
222 & $0.128 \pm 0.023$ & $0.067 \pm 0.018$ & \\
100 & $0.016 \pm 0.001$ & $0.017 \pm 0.002$ & \\
300 & $0.005 \pm 0.001$ & $0.003 \pm 0.001$ & $0.001 \pm 0.0006$ \\
010 & $0.015 \pm 0.001$ & $0.007 \pm 0.003$ & $0.003 \pm 0.001$ \\
220 & $0.067 \pm 0.007$ & & $0.034 \pm 0.005$ \\
002 & $0.067 \pm 0.006$ & & $0.035 \pm 0.005$ \\
221 & & $0.003 \pm 0.002$ & $0.001 \pm 0.0005$ \\
101 & & $0.003 \pm 0.0007$ & $0.001 \pm 0.0008$ \\
302 & & $0.0005 \pm 0.0002$ & $0.0002 \pm 0.0002$ \\
\hline \hline
\end{tabular}

Words with the best state classification in the statistical discriminant analysis.

TABLE II

Results of the Mann-Whitney-U-Test and Discriminant Analysis

\begin{tabular}{llll}
\hline \hline words & p-value & well classified (1) & well classified (2) \\
\hline \multicolumn{3}{l}{ awake (1) vs. sedated(2) } \\
\hline \hline 000 & $<0.0005$ & $100 \%$ & $94.7 \%$ \\
222 & $<0.0005$ & $100 \%$ & $94.7 \%$ \\
100 & 0.003 & $89.5 \%$ & $84.2 \%$ \\
300 & 0.002 & $94.7 \%$ & $84.2 \%$ \\
010 & $<0.0005$ & $100 \%$ & $89.5 \%$ \\
\hline awake (1) vs. anesthetized (2) & \\
\hline 220 & $<0.0005$ & $100 \%$ & $100 \%$ \\
002 & $<0.0005$ & $100 \%$ & $100 \%$ \\
300 & $<0.0005$ & $94.73 \%$ & $100 \%$ \\
010 & $<0.0005$ & $100 \%$ & $100 \%$ \\
\hline sedated (1) vs. anesthetized (2) & \\
\hline 221 & $<0.0005$ & $73.7 \%$ & $94.4 \%$ \\
101 & $<0.0005$ & $78.9 \%$ & $72.2 \%$ \\
302 & $<0.0005$ & $78.9 \%$ & $77.8 \%$ \\
010 & $<0.0005$ & $52.6 \%$ & $100 \%$ \\
\hline \hline
\end{tabular}

\section{B. Analysis of 10-second Windows}

The discriminating words were subsequently used for calculating the probability of these words every 10 s for each patient of the first database.

For validation, the second database was also analyzed with the same method. The $P_{k}$ values achieved for both databases are similar. Several words presented a prediction probability of the effect-site concentration of propofol higher than the one presented by the AAI. In both databases the word " 010 " showed the best ability to reflect the DOA and therefore is presented in Table IV along with the AAI results. 
TABLE III

Results for Shannon and Rényi Entropies

\begin{tabular}{llll}
\hline \hline & Shannon & Rényi q=0.1 & Rényi q=0.15 \\
\hline \hline awk & $4.72 \pm 0.221$ & $5.73 \pm 0.099$ & \\
sdt & $4.48 \pm 0.325$ & $5.59 \pm 0.129$ & $5.51 \pm 0.108$ \\
ansth & $4.16 \pm 0.218$ & $5.44 \pm 0.098$ & $5.34 \pm 0.091$ \\
p-value(awk,sdt) & 0.010 & 0.001 & 0.001 \\
p-value(sdt, ansth) & 0.003 & 0.001 & $<0.0005$ \\
p-value(awk, ansth) & $<0.0005$ & $<0.0005$ & $<0.0005$
\end{tabular}

Mean, standard deviation and statistical significance of the differences between awake (awk), sedated (sdt) and anesthetized (ansth) states.

TABLE IV

Prediction Probability of the Effect-Site-Concentration of Propofol

\begin{tabular}{lllll}
\hline \hline & First database & \multicolumn{3}{l}{ Second database } \\
\hline$P_{k}$ value & word010 & AAI & word010 & AAI \\
\hline \hline mean \pm SD & $0.215 \pm 0.046$ & $0.263 \pm 0.130$ & $0.214 \pm 0.082$ & $0.246 \pm 0.125$ \\
\hline \hline
\end{tabular}

\section{CONCLUSION}

The results show that the symbolic dynamics are able to reflect the depth of anesthesia. In the analysis of the whole EEG, the histograms of each state of anesthesia demonstrates that the probability of the words change according to the depth of anesthesia.

The statistical tests proved that several words are able to discriminate the DOA with a higher percentage than $90 \%$. The Shannon and Rényi entropies of the words for the different states are also able to discriminate the different states but not as well as the selected words and therefore, they were not considered for state characterization.

To create an index for real-time state detection, the probabilities of the words derived from the symbolic dynamics methodology were calculated every 10 s. For evaluating this real time approach, the $P_{k}$ referring to the prediction of the effect-site-concentration of propofol was computed. The results show a slightly better $P_{k}$ value for the probability of word 010 than for the AAI. This confirms the ability of the symbolic dynamics to reflect the DOA. However, it is difficult to interpret why the word 010 reveals the best correlation with the effect-side-concentration. Moreover, the effect-side-concentration is only one of the possible DOA references and so it is advisable to evaluate this application of the symbolic dynamics using other indices of DOA or a clinical evaluation.

This work is a preliminary study and further analysis with more EEG recordings is necessary. Also, the burst suppression periods of the EEG, that reflects a deep level of anesthesia will be considered in the non-linear DOA analysis.

\section{ACKNOWLEDGMENT}

CIBER of Bioengineering, Biomaterials and Nanomedicine is an initiative of ISCIII.

\section{REFERENCES}

[1] A. Vakkuri, "Spectral entropy monitoring is associated with reduced propofol use and faster emergence in propofol-nitrous oxide-alfentanil anesthesia," Anesthesiology, vol. 103, pp.274-9, 2005.

[2] A. Recart, "Effect of auditory evoked potential index monitoring on anesthetic drug requirements and recovery profile after laparoscopic surgery: a clinical utility study," Anesthesiology, vol. 99, pp. 813-8, 2003

[3] L. Jameson, "Using EEG to monitor anesthesia drug effects during surgery," J Clin Monit Comput, vol. 20, pp. 445-7, 2006.

[4] S. Sebel, "The incidence of awareness during anesthesia: A multicenter United States study," Anesth. Analgesia, vol. 99, 2004.

[5] D. Stanski, "Monitoring depth of anesthesia," Anesthesia, New York: Churchill Livingstone, pp.1001-1029, 1990.

[6] E.W. Jensen, "Auto regressive modeling with exogenous input of auditory evoked potentials to produce an on-line depth of anesthesia index," Meth. Inf. Med., vol. 35, pp. 256-260, 1996.

[7] H. Litvan, "Comparison of conventional averaged and rapid averaged, autoregressive-based extracted auditory evoked potentials for monitoring the hypnotic level during propofol induction," Anesthesiology, vol. 97, no. 2, pp. 351-358, 2002.

[8] I.J. Rampil, "A primer for EEG signal processing in anesthesia," Anesthesiology, vol. 89, no. 4, pp. 981-1001, 1998

[9] J. C. Sigl and N. G. Chamoun, "An introduction to bispectral analysis for the EEG," J. Clin. Monit., vol. 10, pp. 392-404, 1994.

[10] E. Başar, "Chaotic dynamics and resonance phenomena in brain function: Progress, perspectives, and thoughts. Chaos in brain function", Springer, Berlin Heidelberg, New York, 1-30, 1990.

[11] A. Voss, "The application of methods of non-linear dynamics for the improved and predictive recognition of patients threatened by sudden cardiac death," Cardiovascular Research, vol. 31, pp. 419-433, 1996.

[12] M. Jospin, "Detrended Fluctuation Analysis of EEG as a Measure of depth of Anesthesia," IEEE Trans. Biomed. Eng., vol. 54. pp. 840846, 2007.

[13] J. Bruhn, "Depth of anesthesia monitoring: what's available, what's validated and what's next?", Br J Anaesth., vol. 97, pp. 85-94, 2006.

[14] T. De Smet and M. Struys [Online]. Available: http://www.anesthesiauzgent.be

[15] T. W. Schnider, C. F. Minto, P. L. Gambus, C. Andresen, D. B. Goodale, S. L. Shafer, and E. J. Youngs, "The influence of method of administration and covariates on the pharmacokinetics of propofol in adult volunteers," Anesthesiology, vol. 88, no. 5, pp. 1170-1182, 1998.

[16] T. W. Schnider, C. F. Minto, S. L. Shafer, P. L. Gambus, C. Andresen, D. B. Goodale, and E. J. Youngs, "The influence of age on propofol pharmacodynamics," Anesthesiology, vol. 90, pp. 1502-1516, 1999.

[17] S. L. Shafer and K. M. Gregg, "Algorithms to rapidly achieve and maintain stable drug concentrations at the site of drug effect with a computer- controlled infusion pump," J. Pharmacokinet. Biopharm., vol. 20, pp. 147-169, 1992.

[18] M. M. R. F. Struys, T. De Smet, B. Depoorter, L. F. Versichelen, E. P. Mortier, F. J. Dumortier, S. L. Shafer, and G. Rolly, "Comparison of plasma compartment versus two methods for effect compartmentcontrolled target-controlled infusion for propofol," Anesthesiology, vol. 92 no. 2, pp. 399-406, 2000.

[19] Hadamard, "Les surfaces à courbures opposées et leurs lignes géodésiques," Journal de Mathématiques Pures et Appliquées, vol. 4, pp. 27-74, 1898.

[20] BL. Hao, "Symbolic dynamics and characterization of complexity," Physica D, vol. 51, pp. 161-176, 1991.

[21] W. Smith, "Measuring the performance of anesthetic depth indicator," Anesthesiology, vol. 84, pp. 38-51, 1996.

[22] A. Vanluchene, "Spectral entropy as an electroencephalographic measure of anesthetic drug effect," Anesthesiology, vol. 101, pp. 34 42, 2004.

[23] M. Koskinen, "Monotonicity of approximate entropy during transition from awareness to unresponsiveness due to propofol anesthetic induction," IEEE Trans Biomed Eng vol. 53, pp. 669-75, 2006.

[24] R. Ellerkmann, "Spectral entropy and bispectral index as measures of the electroencephalographic effects of sevoflurane," Anesthesiology, vol. 101, pp.1275-82, 2004. 